\title{
Syndrome of congenital ventricular diverticulum and midline thoraco-abdominal defects
}

\author{
GIUSEPPE GULA AND MAGDI YACOUB \\ From the Cardiothoracic Surgical Unit, Harefield Hospital, Middlesex
}

Gula, G. and Yacoub, M. (1977). Thorax, 32, 365-369. Syndrome of congenital ventricular diverticulum and midline thoraco-abdominal defects. The long-term follow-up of a 30-year-old patient who had a correction of the syndrome of left ventricular diverticulum and thoraco-abdominal defects is presented. The main features of the syndrome include a diverticulum of the left ventricle, a ventricular septal defect, and sometimes other cardiac anomalies. The thoraco-abdominal defects consist of foreshortened sternum, pericardial and diaphragmatic defects, and umbilical hernia. The association of these anomalies is thought to be due to a developmental failure of the primitive paramidline mesoderm.

The diagnosis can be made clinically by the presence of a pulsatile, epigastric mass associated with signs of cardiac septal defects and dextrocardia. The prognosis of patients with this syndrome depends mainly on the associated abnormalities although rupture of the diverticulum can be a fatal complication.

The treatment of choice is resection of the diverticulum combined with repair of associated anomalies which can give good early and late results.

The association of a congenital ventricular diverticulum with other cardiac abnormalities and midline thoraco-abdominal defects has been described by Cantrell et al. (1958). Its recognition is essential for accurate identification of the different anomalies and their proper treatment. The purpose of this paper is to describe the long-term result in a patient in whom complete correction of the syndrome had been performed six years previously and to draw attention to the characteristic lesions and their significance.

\section{Case report}

A 30-year-old woman was admitted to Harefield Hospital with a history of cyanosis and increasing dyspnoea which were first noted when she was 4 years old after an operation for umbilical hernia. There was no family history of cardiac disease or congenital anomalies. On admission the physical signs included clubbing of the fingers and moderate cyanosis. The jugular venous pressure was normal and the radial pulse regular at a rate of 90 per minute. The blood pressure was $120 / 80 \mathrm{mmHg}$. There was also a slight prominence of the right chest with no definite evidence of ventricular hypertrophy. A pulmonary systolic thrill was felt and a loud systolic murmur was heard in the right parasternal area over the fourth interspace. In the abdomen there was the scar of an umbilical herniorrhaphy but no masses were palpable.

Laboratory tests showed a red cell count of $4 \cdot 3 \times$ $10^{12} / 1$ with a haemoglobin of $18 \mathrm{~g} / \mathrm{dl}$ and haematocrit of $44 \%$. Serological tests for syphilis were negative. The radiograph showed oligaemic lung fields and the heart was slightly enlarged and situated to the right of the spine with the appearance of dextrocardia. However, the aortic knuckle and the stomach were on the left (Fig. 1). The chest wall was asymmetrical with moderate scoliosis. The electrocardiogram showed sinus rhythm at 90 per minute, right axis deviation, an upright $P$ wave in lead 1 , and moderate right ventricular hypertrophy in the chest leads (including the right chest leads). A clinical diagnosis of Fallot's tetralogy with dextroversion was made.

Cardiac catheterisation showed a typical Fallot situation with identical pressures in both ventricles and an atrial septal defect. Angiocardiography showed an anterior ventricle markedly rotated to the right and backwards, giving rise to a pulmonary artery and infundibular and pulmonary 


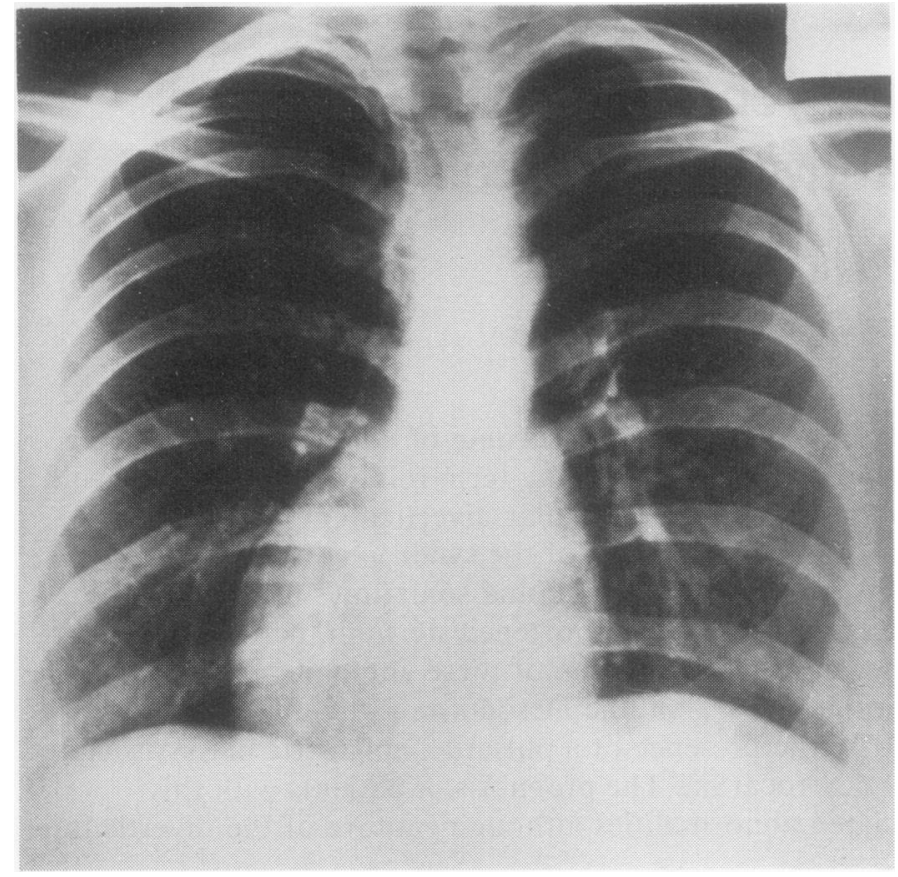

Fig. 1 Posteroanterior chest radiograph showing apparent dextrocardia and oligaemic lung fields. Aortic knuckle and stomach are left sided.

valve stenosis. A tubular diverticulum originating from the posterior ventricle, which was morphologically a left ventricle, was also present. The diverticulum extended downwards and anteriorly in the midline and showed a paradoxical motion, its size increasing more than $100 \%$ during ventricular systole. The contrast medium filled the diverticulum uniformly and there was no evidence of thrombus formation in the sac (Fig. 2).

At operation (August 1970) a foreshortened sternum was found. There was a large conical diverticulum protruding through a defect of the pericardium and diaphragm into the extraperitoneal tissue and extending down to the umbilicus. The diverticulum arose from the apex of the left ventricle and so had caused the dextrorotation of the ventricles. There were several pedicles originating from the abdominal wall and internal mammary vessels attached to the diverticulum (Fig. 3). The pedicles contained venous channels and what looked like nerves, but no large arteries were found. Other findings were typical of Fallot's tetralogy with infundibular and pulmonary valve stenosis. The pulmonary and aortic valves were normally related. Exploration of the right atrium confirmed the presence of a small secundum atrial septal defect. Total correction of the cardiac defects with excision of the tubular diverticulum of the left ventricle was performed on cardio- pulmonary bypass. The pericardial and diaphrag matic defects were also repaired.

The postoperative course was uneventful and the patient was discharged after two weeks. Six years later she remained asymptomatic with evidence of satisfactory correction of the cardiac and chest wall defects.

\section{PATHOLOGY}

The diverticulum was muscular and measured $8 \mathrm{~cm}$ in length with a diameter of $3 \mathrm{~cm}$. The wall was $4 \mathrm{~mm}$ thick, being thinner than the main 의 body of the left ventricle. The internal surface was finely trabeculated, resembling the endocardial surface of the left ventricle (Fig. 4). Histological examination of the sac showed normal myo- o cardial tissue with patchy fibrosis.

\section{Discussion}

True congenital muscular diverticulum of the ventricle is a rare condition. Edgett et al. (1969) $\stackrel{\odot}{+}$ could find only 25 previously reported cases. In the syndrome described, the diverticulum is associated with a group of typical cardiac and $\stackrel{\mathbb{P}}{\stackrel{\oplus}{\circ}}$ thoraco-abdominal defects. A constant feature of $\stackrel{\overrightarrow{\mathbb{Q}}}{\varrho}$ the syndrome is the presence of a ventricular septal defect with or without pulmonary stenosis (Cantrell et al., 1958). Transposition of the great 


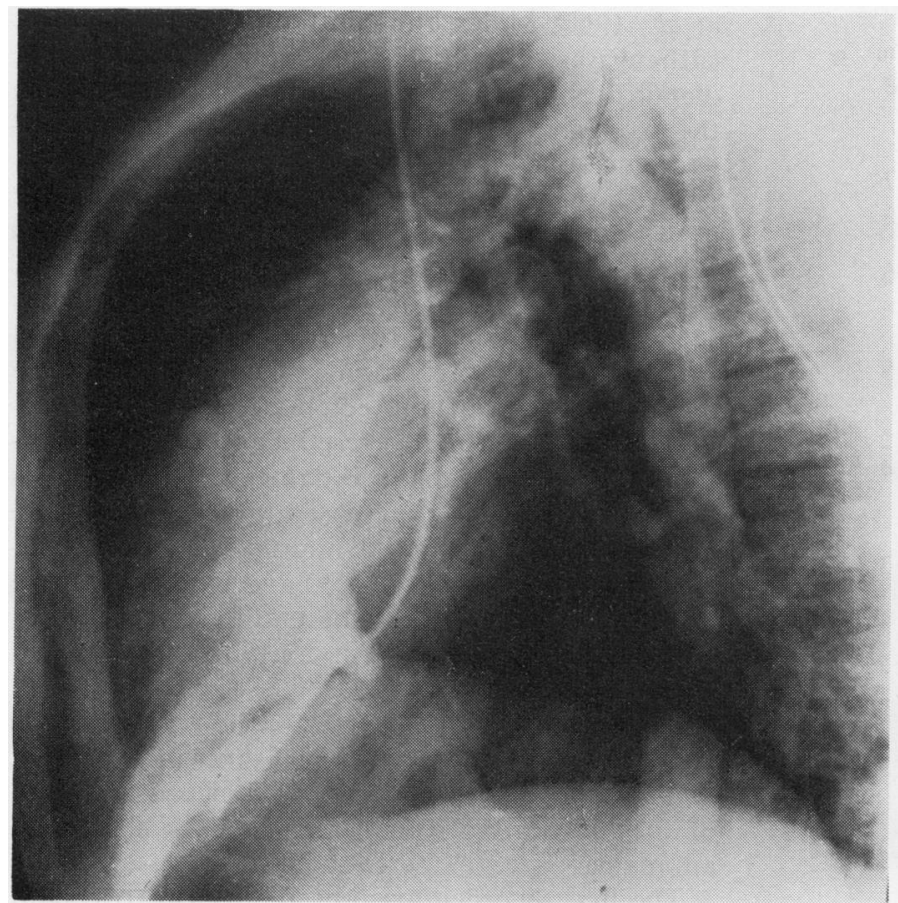

Fig. 2 Selective left ventriculogram (lateral projection) showing a tubular diverticulum filled uniformly by the contrast medium.

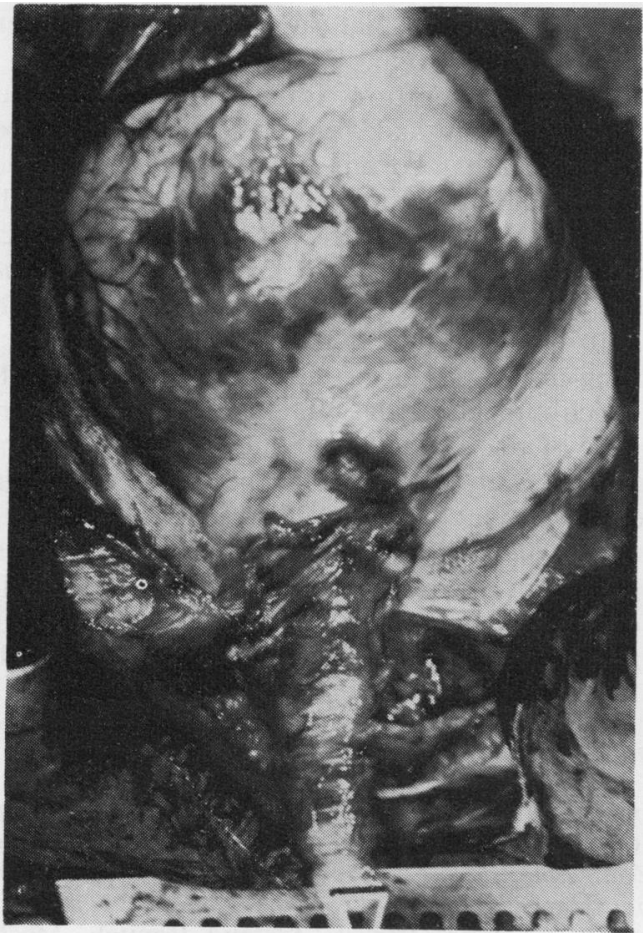

Fig. 3 Intraoperative view of left ventricular diverticulum. arteries, atrial septal defect or atrioventricular canal are very rare additional lesions (Albrecht, 1972; Miller and Matthews, 1973). The diverticulum usually arises from the apex of the left ventricle. The heart therefore rotates in an anticlockwise direction and the apex comes to lie towards the right, thus simulating dextrocardia. However, the aortic knuckle and the other organs are normally situated. Usually the diverticulum leaves the chest through a pericardial and diaphragmatic defect, although sometimes it appears that the pericardium continues to envelop the diverticulum as far as the umbilicus (Lowe $e t$ al., 1959; Mulder et al., 1960; Miller and Matthews, 1973). The pouch of the diverticulum is continuous with the ventricular cavity. Histologically the wall of the diverticulum shows the three layers of the heart with a certain amount of connective tissue. In some cases the main ventricular cavity shows endocardial fibroelastosis (Galindo et al., 1957; Albrecht, 1972). Deformity of the chest wall with a foreshortened sternum can also be present. The defects of the abdominal wall vary from an isolated umbilical hernia to the absence of the anterior segment of the diaphragm, diastasis recti, and an omphalocoele. The association of these anomalies is thought to be due to a developmental failure of the primitive paramidline mesoderm in both its somatic and splanchnic portions. These 


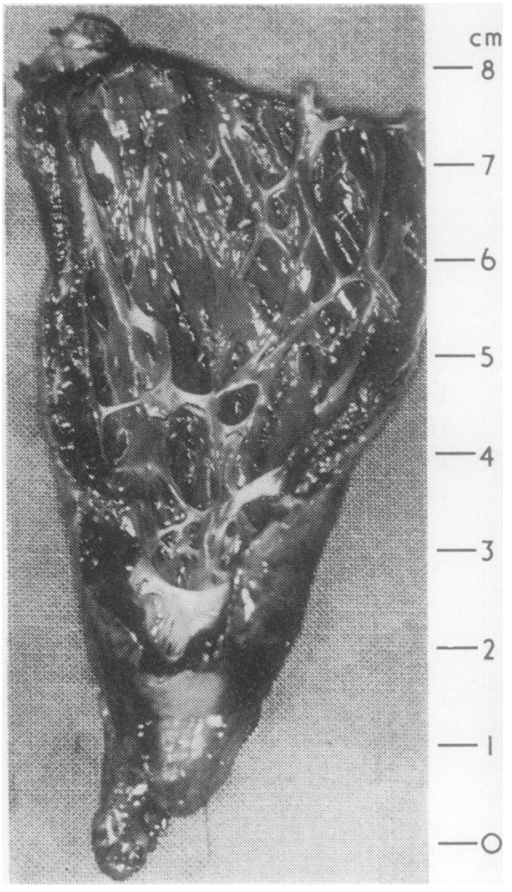

Fig. 4 The resected diverticulum showing trabeculated internal surface with some endocardial fibrosis near the tip.

give rise to the pericardium, heart, diaphragm, and anterior abdominal wall.

A portion of the primitive ventricle may project through the weakened membranous part of the diaphragm as a diverticulum (Cantrell et al., 1958; Treistman et al., 1973).

The diagnosis can easily be made in childhood. The clinical picture is characterised by the presence of a pulsatile epigastric mass which disappears under the sternum, associated with the signs of septal cardiac defects, dextrorotation of the heart, and thoraco-abdominal midline defects. Some patients are first referred to a general surgeon for treatment of an umbilical hernia. The diverticulum can be discovered at that time as a pulsating, epigastric mass situated in the midline of the upper abdomen. In addition, the presence of a scar in the epigastrium, due to previous herniorrhaphy, and situs solitus with dextroversion in a patient with signs of ventricular septal defect or Fallot's tetralogy is diagnostic of the syndrome. Electrocardiographic findings are variable according to the type and severity of the associated cardiac anomalies. A characteristic pattern is the anticlockwise frontal loop due to malrotation of the heart produced by the diverticulum (Mulder et al., 1960; Miller and Matthews, 1973). Cardiac $\stackrel{\overrightarrow{\vec{N}}}{+}$ catheterisation and angiography confirm the diagnosis of ventricular diverticulum and define the associated anomalies as well as the haemodynamic state.

The prognosis of patients with this syndrome appears to be poor as most of the reported cases $\overrightarrow{0}$ died early in life (Edgett et al., 1969; Albrecht, 1972). The causes of death include complications related to the associated cardiac anomalies and rupture of the diverticulum. In the more extreme forms of the syndrome ectopia cordis or omphalocoele can be directly responsible for death because of direct mechanical damage. In some cases rupture is related to increased pressure inside the sac. This haemodynamic finding has been documented by Lowe et al. (1959) and is thought to be due to an obstruction of the mouth of the diverticulum early in systole with continued contraction of its muscular wall.

We believe that whenever the syndrome is diagnosed early assessment and surgical treatment is advisable. The procedure of choice is resection of the sac combined with repair of the associated anomalies.

The first recorded attempt at surgical treatment was by Wieting (1912), who replaced the diverticulum in the pericardial sac and closed the opening in the diaphragm. Edgett et al. (1969), in a review of the literature, found 13 cases in which the diverticulum had been resected, with three operative deaths. To our knowledge, resection of the diverticulum combined with correction of the associated cardiac and thoraco-abdominal defects has been described in two cases (Mulder et al., 1960; Treistman et al., 1973) apart from the patient described in this paper.

Long-term prognosis after excision of the diverticulum depends on the feasibility and efficacy of the correction of the associated cardiac anomalies and the degree of pulmonary vascular disease. Although diffuse fibroelastosis of the left ventricle in such patients has been reported the myocardium appears to be normal in the majority of cases. Six years after the operation our patient is asymptomatic with evidence of anatomically and functionally complete repair of the lesions.

\section{References}

Albrecht, G. (1972). Beitrag zur Morphologie und formalen Genese kongenitaler Herzdivertikel. Zentralblatt für allgemeine Pathologie und pathologische Anatomie, 116, 42-47.

Cantrell, J. R., Haller, J. A., and Ravitch, M. M. (1958). A syndrome of congenital defects involving the abdominal wall, sternum, diaphragm, peri- 
cardium, and heart. Surgery, Gynecology and Obstetrics, 107, 602-614.

Edgett, J. W., Jr., Nelson, W. P., Hall, R. J., Fishback, M. E., and Jahnke, E. J. (1969). Diverticulum of the heart: part of the syndrome of congenital cardiac and midline thoracic and abdominal defects. American Journal of Cardiology, 24, 580-583.

Galindo, L., Areán, V. M., Stevenson, D. S., and Rivera, E. S. C. (1957). Congenital diverticulum of the heart. American Journal of Clinical Pathology, 27, 84-88.

Lowe, J. B., Williams, J. C. P., Robb, D., and Cole, D. (1959). Congenital diverticulum of the left ventricle. British Heart Journal, 21, 101-106.

Miller, J. D. and Matthews, E. C. (1973). Congenital cardiac diverticulum. American Journal of Diseases of Children, 126, 814-816.
Mulder, D. G., Crittenden, I. H., and Adams, F. H. (1960). Complete repair of a syndrome of congenital defects involving the abdominal wall, sternum, diaphragm, pericardium and heart; excision of left ventricular diverticulum. Annals of Surgery, 151, 113-122.

Treistman, B., Cooley, D. A., Lufschanowski, R., and Leachman, R. D. (1973). Diverticulum or aneurysm of left ventricle. American Journal of Cardiology, 32, 119-128.

Wieting, W. (1912). Eine operativ behandelte Herzmissbildung. Deutsche Zeitschrift für Chirurgie, 114, 293-295.

Requests for reprints to: M. H. Yacoub, FRCS, Harefield Hospital, Harefield, Uxbridge, Middlesex. 